\title{
A model for internal photoemission at high-k oxide/silicon energy barriers
}

\author{
O. Engström \\ Microtechnology and Nanoscience, Chalmers University of Technology, SE-412 96 Göteborg, Sweden
}

(Received 1 July 2012; accepted 24 August 2012; published online 25 September 2012)

\begin{abstract}
A model has been developed to describe the emission of electrons from silicon across the oxide energy barrier of metal-oxide-silicon structures. An optical absorption coefficient, exclusively describing the transmission of electrons which are emitted across the barrier, is split from the corresponding experimental quantity for the entire absorption range. This makes it possible to approximate the photo yield in terms of absorption coefficients and density of states without need for explicitly calculated matrix elements of optical transitions. Using this method, theoretical emission yield curves are found in good agreement with measured data. An important conclusion from this work is that values of oxide energy barrier heights should be extracted from different features of the yield data than most often done in the literature. This replaces a commonly used practice for determining the barrier heights, which is shown to be based on optical bulk properties of the silicon crystal. (C) 2012 American Institute of Physics. [http://dx.doi.org/10.1063/1.4754512]
\end{abstract}

\section{INTRODUCTION}

The chase for finding materials with high dielectric constants ( $k$-values) and high energy offset values, $\Delta E$, between the energy bands of insulator/semiconductor combinations is driven by the necessity for continued gate function improvement of metal-oxide-semiconductor (MOS-) transistors. Due to their fundamental importance for the ultimate limit of leakage current, originating from tunneling, the intrinsic quantities, $k$ and $\Delta E$, are basic for the material choice. Hence, the product $k \Delta E$ is a rough navigation aid when choosing new gate insulator candidates. ${ }^{1}$ Additional importance is set by influences from, for example, chemical reactivity, structural stability, and bulk trap concentrations. In this openended process for finding insulator candidates, reliable measurement methods to determine $\Delta E$ are crucial for the continuous development of nanoelectronics.

The dominant techniques for determining insulator/ silicon offset values are X-ray photoelectron spectroscopy (XPS) and internal photoemission spectroscopy (IPE). While XPS to some extent still requires careful attention to charging effects as a result of electron emission from the insulator, ${ }^{2-5}$ IPE seems easier to control from this point of view. ${ }^{6}$ Energy offset values, obtained by IPE for a number of high- $k$ oxides, have been published in the past $\operatorname{decade}^{7-12}$ and detailed reviews of the technique have been released. ${ }^{6,12}$ The majority of measured data in all these cases has followed a mainstream method for establishing $\Delta E$ values, which has settled the barrier value $\Delta E_{C}$ between the conduction bands of silicon and most high- $k$ oxides to a range of $2.0-2.1 \mathrm{eV}$. In the following, it will be demonstrated that the procedure used in these exertions is doubtful.

In a frequently cited work by Powell, theoretical IPE spectra were derived by using simplified density of states distributions. ${ }^{13}$ This led to a cubic yield dependence on photon energy, which was adequate for $\mathrm{SiO}_{2} / \mathrm{Si}$ structures, a tradition still often adhered to also for investigations of high- $k$ based stacks. Following the basic ideas by Powell, an improved model is demonstrated in the present paper for interpretation of IPE data useful also for high- $k$ type of samples. This increases the possible range of barrier values for these film stacks and reveals a more nuanced picture of the physical conditions laying the ground for such experiments.

\section{INTERNAL PHOTOEMISSION}

IPE relies on measuring electric current, generated by optical excitation of charge carriers to energies exceeding an energy barrier and to find the lowest possible photon energy needed for the process. This energy corresponds to the energy offset, $\Delta E$, limiting charge carrier escape. Fig. 1 demonstrates the method for determining band offset values between the semiconductor and the oxide of a MOS structure with n-type silicon. Monochromatic light irradiates the structure from the metal side, passing a semi-transparent metal gate and the oxide into the semiconductor where it excites electron-hole pairs in the semiconductor or in the metal to energy levels such that electrons can pass the barrier. The final level in the excitation process needs to exceed the barrier height to provide the possibility of injection across the oxide. This will give rise to electric current in an external circuit, containing a voltage source for creating appropriate potential differences. These two steps, excitation followed by emission, are the main processes and may be influenced by carrier scattering, barrier lowering by the oxide electric field, charge carrier trapping, tunneling, and by the optical limitations influencing the intensity of the light before reaching the semiconductor. 6

\section{LIGHT ABSORPTION IN SILICON}

When light is absorbed in semiconductor crystals, the concentration of charge carriers created in the energy bands depends on the state densities and the transition probabilities, including the matrix elements for coupling states in the different bands of the Brillouin zone. Since silicon is a semiconductor with indirect bandgap, excitations from the highest valence band state to the lowest conduction band state, 


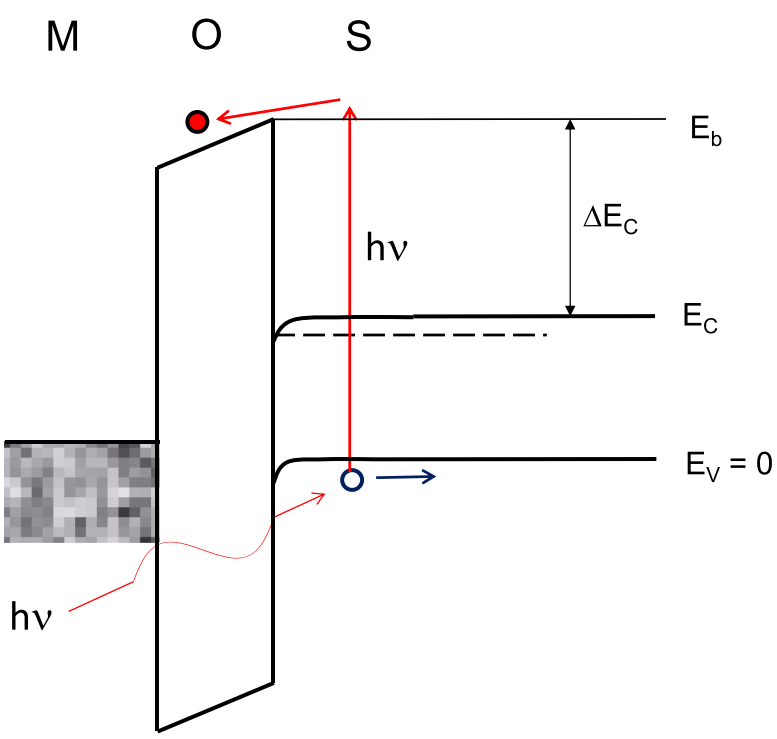

FIG. 1. Energy band diagram of a MOS structure showing the optical excitation of an electron from the silicon valence band to the top of the oxide barrier.

and a couple of electron volts above, also include a coupling between the excited electron and a phonon in order to provide crystal momentum transfer to allow transitions. In addition, direct transitions without the influence of phonon coupling are possible. When interpreting barrier data obtained by IPE on silicon structures, three such energy intervals are of particular interest as featured by numbers (1)-(3) in Fig. 2(a). Transition (1) goes from the valence band to the indirect conduction band within the energy range above the bandgap value, $1.12 \mathrm{eV}$, until the direct transition (2) takes over with a higher intensity. Transition (1) requires electronphonon coupling in order to make a horizontal move in the Brillouin zone. While the direct transition probabilities were elucidated in the $1960 \mathrm{~s},{ }^{14-17}$ transitions of type (1), due to the vast need for computer power, were calculated by first principles only quite recently. ${ }^{18}$ Transitions (1) and (2) are reflected in experimental data for the absorption coefficient, $\alpha$, measured at room temperature by Aspnes and Studna ${ }^{19}$ and shown in Fig. 2(b). Points are measured data tabulated in Ref. 19 and the solid curve is fitted by a combination of analytical expressions to be used in the calculations below. For the lower energies, in the range $2-3.2 \mathrm{eV}$ of this graph, transition (1) is the only possible process and gives rise to an almost exponential increase in $\alpha$ for increasing photon energy. At about $3.2 \mathrm{eV}$ an increased slope is noticed, where the direct transition (2) starts becoming significant, ${ }^{17}$ which results in a saturation of $\alpha$ at about $3.4 \mathrm{eV}$ when this transition passes a maximum probability and transition (3) begins to grow. This latter process may be a direct transition at the X-point in the Brillouin zone from a deeper lying band indicated by the dashed arrow in Fig. 2(a) or from the $\Sigma$ point (not shown in the figure). ${ }^{17,20,21}$ Transition (4) would occur at an energy value close to that of transition (2) and is sometimes mentioned in the literature as a possible contribution to the increasing $\alpha$ trend at $3.2 \mathrm{eV},{ }^{20}$ but its specific importance for IPE results is not clear. Transition (5) is an intra-transition requiring a high concentration of electrons in the conduction
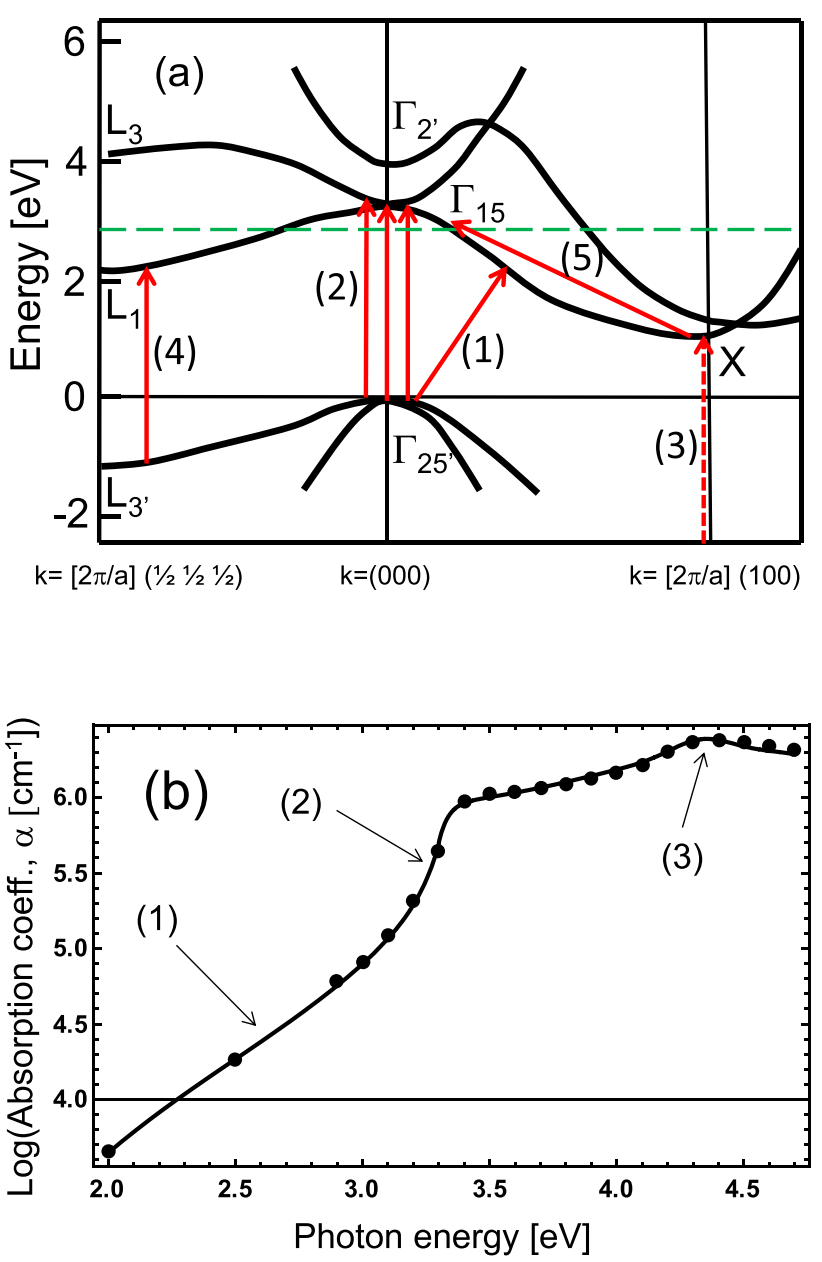

FIG. 2. (a) Optical transitions in the silicon Brillouin zone. (1), (2), and (3) are the most important transitions for giving features to yield spectra of MOS structures. (4) is a transition contributing to emission only for low barriers while (5) is an intra-transition, possibly contributing in strong accumulation. The dashed horizontal line marks a barrier level position. (b) The logarithm of the optical absorption coefficient for silicon as measured by Aspnes and Studna. ${ }^{19}$ The important energy ranges for transitions (1), (2), and (3) shown in (a) are located by the arrows. The solid curve is fitted by a set of piecewise connected analytical functions.

band to be observed. This situation may occur at strong accumulation of an n-type MOS structure as discussed at the end of the paper.

Photons with energy $h \nu$ entering the silicon crystal with an intensity $L_{O}$ and, after absorption, with an endured intensity $L(x)$ at point $x$ measured from the interface would excite $\alpha(h \nu) L(x)$ electron-hole pairs per unit time and volume. For later comparison, we consider an artificial case for $n$-type silicon, where we assume that $\Delta E_{C}=0\left(E_{b}=1.12 \mathrm{eV}\right)$ and that, apart from being killed by scattering with a mean free length $x_{e s c}$, all excited electrons can be emitted from the crystal volume. As $L(x)=L_{0} \exp (-\alpha x)$, the emission yield (the number of emitted electrons per incident photon) would be

$$
Y_{0}(h \nu)=\int_{0}^{\infty} \alpha(h \nu) \exp [-\alpha(h \nu) x] \exp \left[-\frac{x}{x_{e s c}}\right] d x
$$

Using the analytic expression fitted to the experimental data for $\alpha$ in Fig. 2(b) as represented by the solid curve, and using 
Eq. (1), $Y_{O}(h \nu)$ is demonstrated in Fig. 3. Here, we observe that the indirect transitions (1) are very week compared to the two direct excitations (2) and (3). The pronounced step at $3.2 \mathrm{eV}$ is a result of the increasing slope of the absorption coefficient when transition (2) sets in as pointed out above. The curve in Fig. 3 has characteristics very similar to corresponding experimental data measured on bare silicon surfaces as demonstrated in Fig. 7 of Ref. 15. As will be shown below, the feature of this yield curve at $3.2 \mathrm{eV}$ remains when adding a barrier, $\Delta E_{C}>0$. Since it originates from the increasing values of the absorption coefficient for silicon at $3.2 \mathrm{eV}$, it has no connection with oxide barrier heights. IPE data from metal $/ \mathrm{SiO}_{2} / \mathrm{Si}$ structures ${ }^{22}$ and, as will be discussed next, from high- $k$ based MOS structures show a similar ledge at this energy.

\section{EMISSION ACROSS THE OXIDE BARRIER}

In order to treat the emission problem in the presence of an oxide barrier, we consider the energy band scheme shown in Fig. 4(a). This figure illustrates that excited electrons available for emission across the oxide barrier resulting from photons of energy $h \nu$ are not caused by all available absorption events for such excitations. Electrons transmitted to levels above $E_{b}$ have their initial states within an interval $E_{b}-h \nu<E<E_{V}$ and the final states within $E_{b}<E<E_{V}$ $+h \nu$. On the other hand, the transitions governing the absorption coefficient, $\alpha(h \nu)$, span a larger interval given by $h \nu-\Delta E_{G}$. Therefore, among photons with $h \nu$ larger than $E_{b}$, there is a certain fraction that have their initial states deep enough in the valence band that their final states are below the barrier level (dashed arrow in Fig. 4(a)). Those photons are absorbed without contributing to the emission. The total number of absorption events expressed by the coefficient $\alpha(h \nu)$, only produces a fraction of electrons at final states which gives rise to carrier emission. A rigorous solution of this problem, to find an absorption coefficient for transitions leading to emission, would require a first principle calculation similar to the one by Noffsinger et al. ${ }^{18}$ but for transitions with final states above $E_{b}$ only.

In order to find a more comfortable technique, we consider an approximation, where we split the experimental

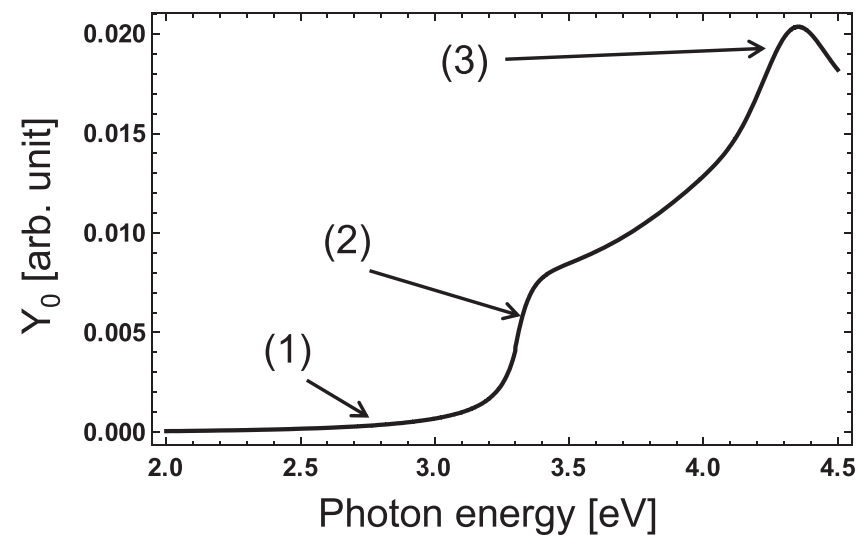

FIG. 3. Yield curve for an artificial structure with $\Delta E_{C}=0$. The influences from the three main transitions (1), (2), and (3), marked in Fig. 2, identify the features of the curve. (a)

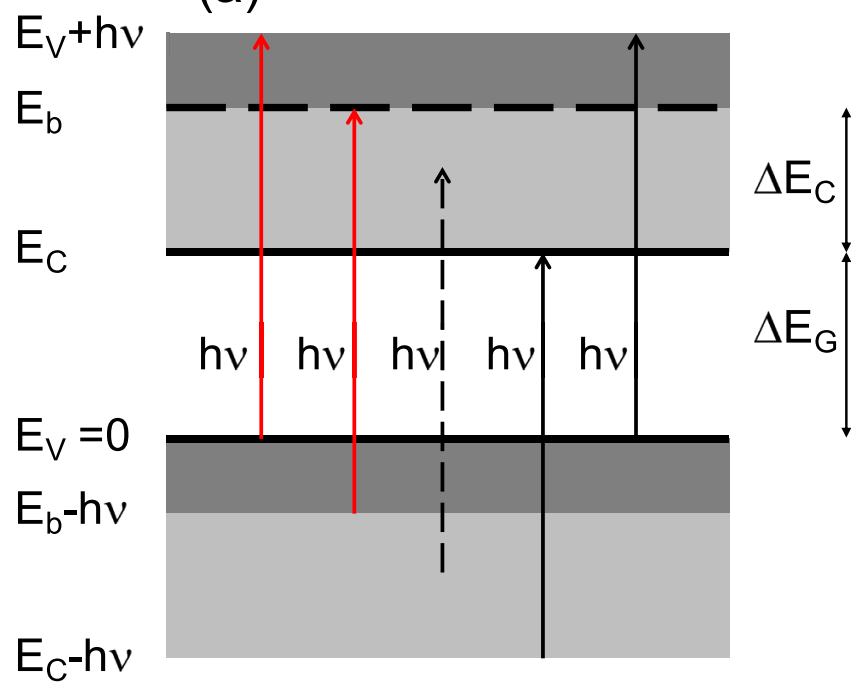

FIG. 4. (a) Energy band diagram showing possible energy ranges for excitation of electrons from the valence band to the conduction band by a photon of energy $h \nu$. The red arrows denote the excitation range leading to emission, the black solid arrows point out the energy range of all possible absorption events. The dashed black arrow demonstrates that, depending on the energy position of the initial state, there are photons with energy $h \nu$, which do not give rise to emission. (b) Density of states for silicon as calculated by Stukel and Euwema ${ }^{23}$ (points), fitted by a set of piecewise connected analytical functions (solid curves).

absorption coefficient, $\alpha$ shown in Fig. 2(b), into one factor, $A(h \nu)$ describing the influence of transition probabilities and a second part, $\gamma(h \nu)$, based on the density of states, $D_{V}$ and $D_{C}$ for the silicon valence and conduction bands, respectively

$$
\alpha(h \nu)=A(h \nu) \gamma(h \nu) .
$$

The function $\gamma(h \nu)$ is the convolution of $D_{V}(E-h \nu) D_{C}(E)$ taken across the same energy interval as that involved in the transitions governing the experimentally obtained $\alpha(h \nu)$, i.e., from the conduction band edge to the maximum energy reached by $h \nu$

$$
\gamma(h \nu)=\int_{E_{C}}^{h \nu} D_{V}(E-h \nu) D_{C}(E) d E .
$$


Knowing $\alpha(h \nu)$ and $\gamma(h \nu)$ would give a possibility to calculate $A(h \nu)$ and achieve an absorption coefficient, exclusively describing transitions with final states high enough for facilitating emission. This can be done by repeating the integral in Eq. (3), but starting the integration from $E_{b}$ and multiplying the result by $A(h \nu)$.

Density of states data for silicon has been calculated by Stukel and Euwema ${ }^{23}$ as reproduced by the points in Fig. 4(b). The solid curves in the figure have been fitted by a combination of analytic functions for use in Eq. (3). Exploiting $\gamma(h \nu)$ achieved by using those data in Eq. (2) and the corresponding analytical expressions for $\alpha(h \nu)$, given by the solid curve in Fig. 2(b), $A(h \nu)=\alpha(h \nu) / \gamma(h \nu)$ gives a measure on the influence of the optical transition probabilities, from which an absorption coefficient, $\alpha_{b}(h \nu)$, for transitions exclusively leading to emission can be calculated

$$
\alpha_{b}(h \nu)=A(h \nu) \int_{E_{b}}^{h \nu} P(E) D_{V}(E-h \nu) D_{C}(E) d E .
$$

The function $P(E)$ in this expression is the probability for an electron in the silicon crystal with an energy above the oxide barrier level, $E_{b}$, to enter the oxide conduction band. This is specified by the "escape cone" and given by ${ }^{6,13}$

$$
P(E)=\frac{1}{2}\left[1-\left(\frac{E_{0}+E_{b}}{E_{0}+E}\right)^{1 / 2)}\right] .
$$

Here, $E_{0}(\approx 12 \mathrm{eV})$ is the kinetic energy in relation to the bottom of the valence band for an electron at the barrier level, $E_{b}$, and $E$ is the energy of the final state of an excitation to the conduction band. ${ }^{13}$

Eq. (4) can be used to calculate the emission yield $Y(h \nu)$, for carriers excited to energy levels above $E_{b}$, by realizing that the production rate of that carrier concentration is $L_{0} \alpha_{b}(h \nu) \exp [-\alpha(h \nu) \mathrm{x}]$

$$
Y(h \nu)=\int_{0}^{\infty} \alpha_{b}(h \nu) \exp [-\alpha(h \nu) x] \exp \left[-\left(\frac{x}{x_{e s c}}+\frac{z_{m}}{z_{0}}\right)\right] d x .
$$

Here again, $x_{\text {esc }}$ is the escape length for electron-electron scattering in the silicon crystal, while the quantity $z_{m}$ is the distance from the silicon interface to the maximum point of the oxide conduction band as a result of barrier lowering and $z_{0}$ is the scattering length of electrons in the image force well at the silicon/oxide interface. ${ }^{24}$

Fig. 5 shows an example of yield curves calculated from Eq. (6) for oxide voltage drops between 0.1 and $4 \mathrm{~V}$ of a MOS structure with a barrier, $E_{b}=2.9 \mathrm{eV}\left(\Delta E_{C}=2.9\right.$ $-1.1=1.8 \mathrm{eV}$ ), physical thickness of $2 \mathrm{~nm}$, dielectric constant equal to 15 , and by taking into account barrier lowering in the oxide as a result of the applied voltage. Following standard procedure in the literature, we plot $Y^{1 / 3}(h \nu)$ for comparison. We notice that the increased silicon absorption at a photon energy of about $3.2 \mathrm{eV}$ gives a pronounced ledge also in these yield curves and that tails occur at lower photon energies. This part of the yield curves indicates the barrier

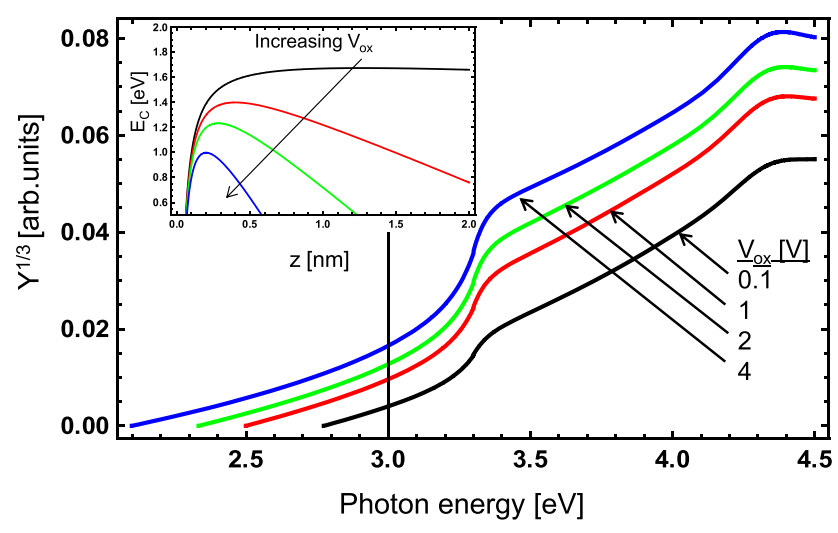

FIG. 5. Calculated yield curves using Eq. (6) for a MOS structures on n-type silicon with an oxide thickness of $2 \mathrm{~nm}, k=15, E_{b}=2.9 \mathrm{eV}$. The inset shows the influence on the oxide conduction band for different oxide voltage drops from 0.1 to $4 \mathrm{~V}$ with the same intervals as for the main figure. $x_{e s c}=8.5 \mathrm{~nm}$ and $z_{0}=6.5 \mathrm{~nm}$ were used.

height by its intersection with the abscissa axis, moving towards lower photon energies due to the barrier lowering as demonstrated in the inset of Fig. 5. The maximum point of the oxide conduction band, which constitutes the oxide barrier for an applied oxide voltage, is obtained by calculating the electrostatic force acting on an escaping electron as a result of mirror effect in the silicon crystal. ${ }^{6}$ This gives the following dependence of $E_{b}$ on the oxide voltage, $V$ :

$$
E_{b}=E_{b 0}-q\left(\frac{q}{4 \pi \varepsilon_{i} \varepsilon_{0}}\right)^{1 / 2}\left(\frac{V_{o x}}{d}\right)^{1 / 2}
$$

where $E_{b 0}$ is the barrier position for $V_{o x}=0, q$ is the electron charge, $\varepsilon_{i}$ is the electronic part of the dielectric constant of the oxide, $\varepsilon_{0}$ is the permittivity of free space, and $d$ is the physical thickness of the oxide. Using Eq. (7) in Eq. (4) to calculate $E_{b}$ for different voltage values, the influence of voltage on the yield curves was achieved as shown in Fig. 5. The effect also contributes to the increased yield amplitudes for increasing gate voltage, together with the shift in barrier maximum position, also recognized in the inset. The latter appearance decreases the value of $z_{m}$ in Eq. (6), which has an influence on the scattering in the oxide image force well.

It is obvious that when determining barrier heights from the zero point of $Y^{1 / 3}(h \nu)$ data, one needs to take into account the strong influence by the barrier lowering. In practice, this is done by extrapolating barrier values obtained at different voltages to $V_{o x}=0 .{ }^{6}$ However, not seldom the ledge at $3.2 \mathrm{eV}$ in $Y^{1 / 3}(h \nu)$ is taken as a result of emission across the barrier. ${ }^{6-12}$ It is clear from the derivation above that this feature of yield curves on silicon samples originates from the $\Gamma_{25},-\Gamma_{15}$ transition labeled (2) in Figs. 2 and 3 and not from a barrier ascent.

Fig. 6 shows a comparison between calculated yield and experimental data from measurements on rare-earth scandates and an aluminate with $20 \mathrm{~nm}$ physical thickness and an applied electric field across the oxide of $2 \mathrm{MV} / \mathrm{cm}^{12}$ The experiments showed a very similar appearance of $Y^{1 / 3}(h \nu)$ for $\mathrm{LaScO}_{3}, \mathrm{GdScO}_{3}, \mathrm{DyScO}_{3}$, and $\mathrm{LaAlO}_{3}$ and the experimental points in the figure are replotted from the middle of the merging experimental points for these oxides. In Ref. 12 


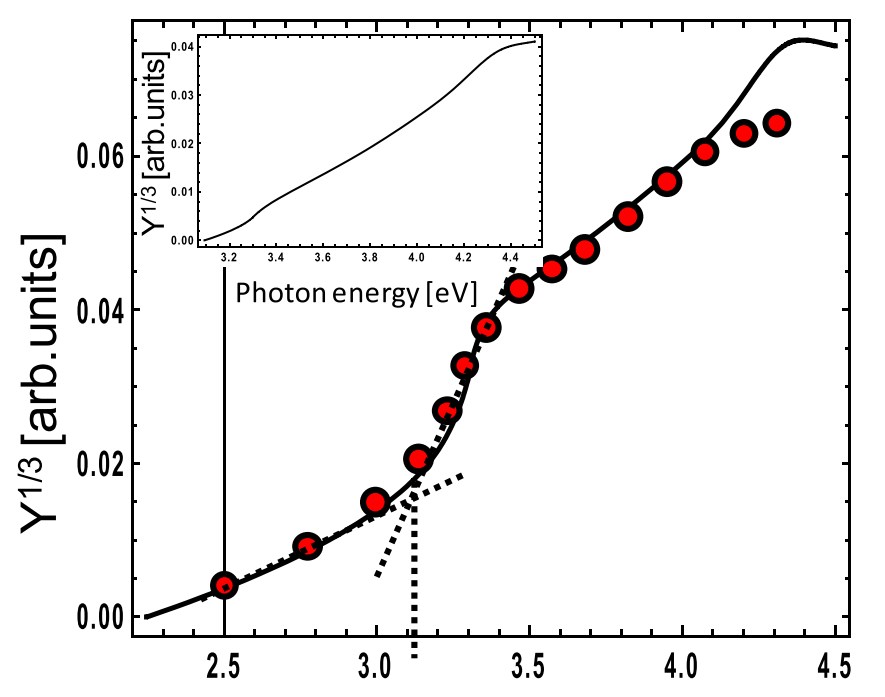

Photon energy [eV]

FIG. 6. Comparison between measured yield data from Ref. 12 (points) and calculated values from Eq. (6) (solid curve) for rare-earth scandates. The dashed lines mark the energy position at $3.1 \mathrm{eV}$ interpreted as the barrier height in Ref. 12. The tail of the curve points at $E_{b}=2.25 \mathrm{eV}$, obtained by using an oxide field of $2 \mathrm{MV} / \mathrm{cm}$ and for a thickness of $20 \mathrm{~nm}$. The zerovoltage value of $E_{b}$ was assumed $2.5 \mathrm{eV}$ in the calculation. Inset: Same calculation as for the main graph assuming a barrier height of $3.1 \mathrm{eV}$.

(Fig. 17), a barrier height of $E_{b}=3.1 \mathrm{eV}\left(\Delta E_{C}=3.1-1.1\right.$ $=2.0 \mathrm{eV}$ ) was extracted from these data, by referring to the position of the absorption ledge, discussed above. The solid curve in Fig. 6 is calculated for the same physical data, but with a barrier height of $E_{b 0}=2.5 \mathrm{eV}$, meaning that $\Delta E_{C}=1.4 \mathrm{eV}$. The abscissa intersection at $2.25 \mathrm{eV}$, lower than $E_{b 0}$, is a result of barrier lowering. The agreement between measured and calculated data suggests that the tail in the yield spectrum gives the barrier height, while the ledge at $3.1 \mathrm{eV}$ is a result of the $\Gamma_{25},-\Gamma_{15}$ transition in the silicon bulk. The difference between the calculated and measured data for photon energies above $4 \mathrm{eV}$ may be caused by problems to calibrate the photon intensity reaching the silicon crystal for MOS sample variations of the semitransparent metal thickness. The inset of Fig. 6 shows a calculated result expected for a measurement on a structure with $E_{b}=3.1 \mathrm{eV}$, unveiling a spectral shape dissimilar to the measured data.

\section{DISCUSSION}

The structure above $3.2 \mathrm{eV}$ in the yield curves is entirely a result of the features of the absorption coefficients, $\alpha$ and $\alpha_{b}$, which is realized from their roles in Eq. (6). On the other hand, the complicated shape of the density of states in the silicon conduction band, as seen in Fig. 4(b) for energies above $2 \mathrm{eV}$, has a much smaller influence on the detailed shape of $Y$. The reason is the integrations done in Eqs. (3) and (4), which smooth out the peaks in $D_{C}(E)$. We can conclude, therefore, that the ledge at $3.2 \mathrm{eV}$ in the yield curves of Figs. 3, 5 and 6 in all cases originate from the $\Gamma_{25},-\Gamma_{15}$ transition (Fig. 2(a)) and the increased slope at $3.2 \mathrm{eV}$ in the logarithmic plot of $\alpha$ which has no connection with the oxide barrier. The technique described for deriving Eqs. (2)-(6) above, by using the ratio between the experimental data for the absorption coefficient, $\alpha$ (Fig. 2(b)) and the convolution between density of states functions (Fig. 4(b), Eq. (3)) in order to achieve the influence of the transition probabilities, including the optical matrix elements, is found to produce a good agreement between calculated yield results and experimental data as shown in Fig. 6.

Considering the inset of Fig. 5, one notices that for this oxide, the barrier may become very thin just below the barrier maximum. For $V_{o x}=2 \mathrm{~V}$, the barrier lowering gives rise to $\Delta E_{C} \approx 1.2 \mathrm{eV}$. However, an electron excited to $1 \mathrm{eV}$ in the silicon conduction band will have a tunneling distance of about $0.6 \mathrm{~nm}$ to pass into the oxide conduction band. Therefore, in experiments, tunneling cannot be excluded as an additional cause for moving the interception point between the tail in the yield curve and the abscissa to lower values of the photon energy. From a plot of the measured barrier height as a function of $V^{1 / 2}$, a straight line is expected as long as barrier lowering expressed by Eq. (7) is the only origin of an energy shifted yield curve. For an additional tunneling effect, the negative slope of such a plot (Eq. (7)) should increase with increasing voltage. Furthermore for gate voltages high enough, a considerable concentration of electrons would occur by accumulation in the conduction band close to the oxide/silicon interface, which may give rise to intra-transitions within the conduction band labelled (5) in Fig. 2(a), a possible effect suggested in Ref. 15. Depending on the concentration of accumulated electrons, an added tail in the yield spectra might occur with a threshold value at $E_{b}-1.1 \mathrm{eV}$. Such band bending in accumulation also gives rise to a lowering of the oxide barrier level in relation to the energy band edges deeper into the semiconductor as a result of the applied bias. The depth of this accumulation region may be of the same extent as the carrier scattering length and the optical absorption depth and influence measured results. ${ }^{24}$ All these effects would increase the tail of the yield curve and give rise to a further decrease of the interception point with the abscissa of a yield plot, which motivates the execution of measurements to be performed for varied applied voltage across the MOS structure.

By deriving a model for internal photoemission in silicon MOS structures, it has been demonstrated that the ledge in the emission yield curves occurring at $3.2 \mathrm{eV}$, which is often interpreted as the oxide barrier height in published data ${ }^{6-12}$ on silicon MOS structures with high- $k$ oxides, originates from optical absorption in the silicon bulk. Barrier height values for $E_{b}<3.2 \mathrm{eV}$ are to be extracted from the low energy tails of yield spectra after considering the influence of barrier lowering due to the influence of oxide electric field.

${ }^{1}$ O. Engstrom, B. Raeissi, S. Hall, O. Buiu, M. C. Lemme, H. D. B. Gottlob, P. Hurley, and K. Cherkauoi, Solid-State Electron. 51, 622 (2007).

${ }^{2}$ R. Puthenkovilakam and J. P. Chang, J. Appl. Phys. 96, 2701 (2004)

${ }^{3}$ E. Bersch, M. Di, S. Consiglio, R. D. Clark, G. J. Leusink, and A. C. Diebold, J. Appl. Phys. 107, 043702 (2010).

${ }^{4}$ M. Perego and G. Seguino, J. Appl. Phys. 110, 053711 (2011).

${ }^{5}$ I. Z. Mitrovic, S. Hall, N. Sedghi, G. Simutis, V. R. Dhanak, P. Bailey, T. C. Q. Noakes, I. Alexandrou, O. Engstrom, J. M. J. Lopes, and J. Schubert, J. Appl. Phys. 112, 044102 (2012). 
${ }^{6}$ V. V. Afanas'ev, Internal Photoemission Spectroscopy, Principles and Applications (Elsevier, Amsterdam, 2008).

${ }^{7}$ V. V. Afanas'ev, M. Houssa, A. Stesmans, and M. M. Heyns, Appl. Phys. Lett. 78, 3073 (2001); J. Non-Cryst. Solids 303, 69 (2002).

${ }^{8}$ V. V. Afanas'ev, A. Stesmans, C. Zhao, M. Caymax, T. Heeg, J. Schubert, Y. Jia, D. G. Schlom, and G. Lucovsky, Appl. Phys. Lett. 85, 5917 (2004).

${ }^{9}$ M. Badylevich, S. Shamuilla, V. V. Afanas'ev, A. Stesmans, A. Laha, H. J. Osten, and A. Fissel, Appl. Phys. Lett. 90, 252101 (2007).

${ }^{10}$ M. Lei, J. H. Yum, S. K. Banrejee, G. Bersuker, and M. C. Downer, Phys. Status Solidi B 249, 1160 (2012).

${ }^{11}$ G. Seguini, E. Bonera, S. Spiga, G. Scarel, and M. Fanciulli, Appl. Phys. Lett. 85, 5316 (2004)

${ }^{12}$ V. V. Afanas'ev and A. Stesmans, J. Appl. Phys. 102, 081301 (2007).

${ }^{13}$ R. J. Powell, J. Appl. Phys. 41, 2424 (1970).
${ }^{14}$ G. W. Gobeli and F. G. Allen, Phys. Rev. 127, 141 (1962).

${ }^{15}$ F. G. Allen and G. W. Gobeli, Phys. Rev. 144, 558 (1966).

${ }^{16}$ E. O. Kane, Phys. Rev. 127, 131 (1962).

${ }^{17}$ D. Brust, Phys. Rev. 139, A489 (1965).

${ }^{18}$ J. Noffsinger, E. Kioupakis, C. G. Van de Walle, S. G. Louie, and M. L. Cohen, Phys. Rev. Lett. 108, 167402 (2012).

${ }^{19}$ D. E. Aspnes and A. A. Studna, Phys. Rev. B 27, 985 (1983).

${ }^{20}$ D. Brust, M. L. Cohen, and J. C. Phjillips, Phys. Rev. Lett. 9, 389 (1962).

${ }^{21}$ J. R. Chelikowsky and M. L. Cohen, Phys. Rev. B 10, 5095 (1974).

${ }^{22}$ C. R. Wiswanathan and S. Ogura, Phys. Rev. B 1, 624 (1970).

${ }^{23}$ D. J. Stukel and R. N. Euwema, Phys. Rev. B 1, 1635 (1970).

${ }^{24}$ H. Przewlocki, D. Brzezinska, and O. Engstrom, J. Appl. Phys. 111, 114510 (2012). 